\title{
Definindo um Sistema de Monitoramento em Tempo real para o Transporte Público da Cidade de Manaus
}

\author{
Matheus de Sousa Brito \\ Instituto Federal do Amazonas - IFAM \\ Manaus, AM, Brasil \\ 2019004705@ifam.edu.br \\ Gabriel Fortes Marques \\ Instituto Federal do Amazonas - IFAM \\ Manaus, AM, Brasil \\ 2019004607@ifam.edu.br
}

\author{
Diego Bruzaca de Souza \\ Instituto Federal do Amazonas - IFAM \\ Manaus, AM, Brasil \\ 2019004670@ifam.edu.br
}

\author{
Priscila Silva Fernandes \\ Instituto Federal do Amazonas - IFAM \\ Manaus, AM, Brasil \\ priscila.fernandes@ifam.edu.br
}

\author{
Luís Victor Bastos Rezende \\ Instituto Federal do Amazonas - IFAM \\ Manaus, AM, Brasil \\ 2019001641@ifam.edu.br
}

\author{
Vitor Bremgartner da Frota \\ Instituto Federal do Amazonas - IFAM \\ Manaus, AM, Brasil \\ vitorbref@ifam.edu.br
}

\author{
Luis Rivero \\ PPGCC/UFMA \\ São Luis, MA, Brasil \\ luis.rivero@ufma.br
}

\begin{abstract}
The management of public transportation suffers from the difficulty of predicting population growth and consequently meet its demands in terms of quality and safety. With regard to safety, several computer systems have emerged to help prevent and treat transport problems, such as detour of routes and speeding. However, in the context of the Manaus city, these systems cannot: (a) detect specific objects (firearms, knives, among others) to identify assaults; and (b) identify overcrowding in means of transportation. The inclusion of these functionalities with the above mentioned ones may allow access to more information to subsidize decision making and improve the quality of security offered by the public transportation service. This article presents a proposal for an intelligent system to monitor the safety of a bus related to the above mentioned points. In order to raise the requirements and propose the graphical interface of the system, the techniques of benchmarking and focus group were used. The prototype presented allows simulating how the information would be presented to those responsible for monitoring the bus, in addition to generating reports for monitoring the history of the means of transport and to support decision making regarding the safety of passengers and servers.
\end{abstract}

\section{PALAVRAS-CHAVE}

Gestão, Transporte, Segurança, Qualidade, Benchmarking

\section{INTRODUÇÃO}

Um dos grandes problemas que as metrópoles e megalópoles enfrentam inerentes ao transporte público é em seu processo de gerenciamento. No contexto da cidade de Manaus, pelo grande crescimento demográfico ocorreu a expansão da área urbana como destacou Costa [1]: "Manaus cresceu muito nas últimas quatro décadas, principalmente devido à zona franca e ao distrito industrial. [...] $\mathrm{Na}$ formação social manauara, o poder público, historicamente, não teve estrutura ou capacidade para interferir no processo de urbanização e de ordenar o espaço urbano em sua totalidade”. Todos esses fatos contribuíram negativamente suscitando assim certa dificuldade em prever situações diversas nos ônibus como: superlotações, furtos, desvios de rota, veículos em alta velocidade, etc. Além desses fatos irrefutáveis, o crescimento populacional requer um aumento significativo na demanda de transporte público, devido ao número de pessoas, utilizando o mesmo, ser maior e dessa maneira tornando-se mais árduo o gerenciamento [2].

Uma forma de solucionar tais problemas, é inserindo nos meios de transporte público um sistema de monitoramento em tempo real interligado a uma central de gerenciamento do tráfego urbano no órgão responsável pelo transporte público, levando em consideração que não há atualmente sistemas voltados diretamente para essa área. Esse sistema poderá identificar e auxiliar na solução de problemas referidos anteriormente e dessa forma proporcionar mais qualidade e segurança aos passageiros e colaboradores.

\section{SAFE BUS}

\subsection{Metodologia de Concepção da Proposta}

Para a busca de requisitos, uma das técnicas utilizadas foi a técnica de benchmarking, que consiste em um processo de pesquisa entre empresas (ou sistemas no caso utilizado) de mesma categoria para analisar como seus produtos, processos e serviços estão desempenhando em relação aos concorrentes [3]. Para aplicar essa técnica foi necessário investigar quem eram os atores e seus objetivos, assim como os sistemas equivalentes para tomar como referência e verificar os requisitos e possíveis melhorias.

A outra metodologia utilizada foi a técnica de grupo focal, que se baseia em reunir um grupo pequeno de indivíduos e extrair as opiniões individuais a respeito da problemática discutida [4]. Os principais pontos levantados para a discussão eram ideias que abordavam sobre a segurança, rotas, superlotação, dentre outras situações dos ônibus na cidade de Manaus, dessa maneira possibilitando a construção de requisitos para o sistema de acordo com as seguintes etapas:

1 - Na primeira etapa foi reunido um grupo de participantes e os próprios membros da equipe para discutir sobre a situação dos ônibus da cidade de Manaus, foram relatadas as experiências 
vividas por cada indivíduo; temas como assaltos e excesso de pessoas nos ônibus foram os assuntos mais frequentes e debatidos. Por conseguinte, foram exploradas ideias sobre como o sistema de monitoramento deveria agir em cada situação.

2 - Depois do levantamento de informações o grupo analisou o que foi relatado, dessa maneira foram obtidos os dados necessários para desenvolver os requisitos. Informações como chegada dos ônibus nas paradas ou manutenção, foram algumas das ideias descartadas, visto que foram consideradas ideias que não são adequadas para o conceito central do sistema.

\subsection{Os requisitos}

Após o uso dessas duas técnicas foi elaborada uma tabela com os requisitos funcionais, não funcionais e inversos propostos para o sistema. Dentre esses, os principais dispõem-se abaixo, na Tabela 1.

Tabela 1: Principais requisitos

\begin{tabular}{|l|l|}
\hline ID & Requisitos \\
\hline$[R F 01]$ & O sistema deve detectar o fluxo de passageiros \\
\hline$[R F 02]$ & O sistema deve detectar assalto \\
\hline$[R F 03]$ & O sistema deve detectar desvios de rotas não planejadas \\
\hline$[R F 04]$ & O sistema deve ser capaz de gerar um relatório \\
\hline$[R N F 01]$ & $\begin{array}{l}\text { O sistema deve detectar se o veículo ultrapassar a velocidade máxima determinada } \\
\text { por cada trajeto }\end{array}$ \\
\hline$[$ RNF02] & O sistema deve emitir um alerta em menos de 20s quando detectar um assalto \\
\hline$[R N F 03]$ & O sistema deve alertar sobre um desvio de rota de mais de $500 m$ \\
\hline$[R N F 04]$ & O sistema deve detectar o número máximo de pessoas \\
\hline$[R I 01]$ & O sistema não pode funcionar com o GPS desligado \\
\hline$[R I 02]$ & O sistema não pode se desconectar da rede por mais de 30 minutos \\
\hline
\end{tabular}

\subsection{Concepção do sistema}

O sistema será desenvolvido em linguagem $C$, uma vez que tem uma boa portabilidade em diferentes tipos de sistemas, além de possuir recursos de baixo nível e também ser uma linguagem estruturada. Para Schildt, "A característica especial de uma linguagem estruturada é a compartimentalização do código e dos dados. Trata-se da habilidade de uma linguagem seccionar e esconder do resto do programa todas as informações necessárias para se realizar uma tarefa específica" [5]. No que tange ao rastreamento visual dentro do transporte público, uma das abordagens que atualmente é bastante utilizada é o uso de redes neurais convolucionais, que apresenta bons resultados em sua implementação.

Como descreveu Vargas [6] "Uma rede neural convolucional consiste em múltiplas partes com funções diferentes. [...] Uma camada de convolução é composta por diversos neurônios, cada um responsável por aplicar um filtro em um pedaço específico da imagem. Podemos imaginar cada neurônio sendo conectado a um conjunto de pixels da camada anterior e que a cada uma dessa conexões se atribui um peso. A combinação das entradas de um neurônio, utilizando os pesos respectivos de cada uma de suas conexões, produz uma saída passada para a camada seguinte. Os pesos atribuídos às conexões de um neurônio podem ser interpretados como uma matriz que representa o filtro de uma convolução de imagens no domínio espacial [...]”.

Existem alguns algoritmos de treinamento disponíveis na biblioteca OpenCV [6, 7] e alguns métodos como Features de Haar com AdaBoost para a detecção de rostos e também o descritor HOG e classificador SVM no reconhecimento de poses humanas em imagens de profundidade. Assim sendo, o uso dessas técnicas poderá proporcionar a detecção dos passageiros, assim como suas ações.

\section{A PROTOTIPAÇÃO DA INTERFACE}

Por meio de um benchmarking e um grupo focal foram definidas funcionalidades para a prototipação do sistema que abrangem as seguintes características: A visualização e livre manipulação do mapa da cidade de Manaus em três formas diferentes (modo mapa, modo satélite, modo híbrido); pesquisa de ônibus; gerar relatórios; visualizar um gráfico referente a quantidade geral ou especifica de ônibus e também de pessoas; verificar a integridade de sensores; verificar o fluxo de passageiros por meio do uso de visão computacional; verificar furtos; verificar desvios de rotas não previstos; verificar a velocidade dos ônibus e; permitir a visualização em tempo real das câmeras dentro dos ônibus. A Figura 1 ilustra a interface inicial do sistema.

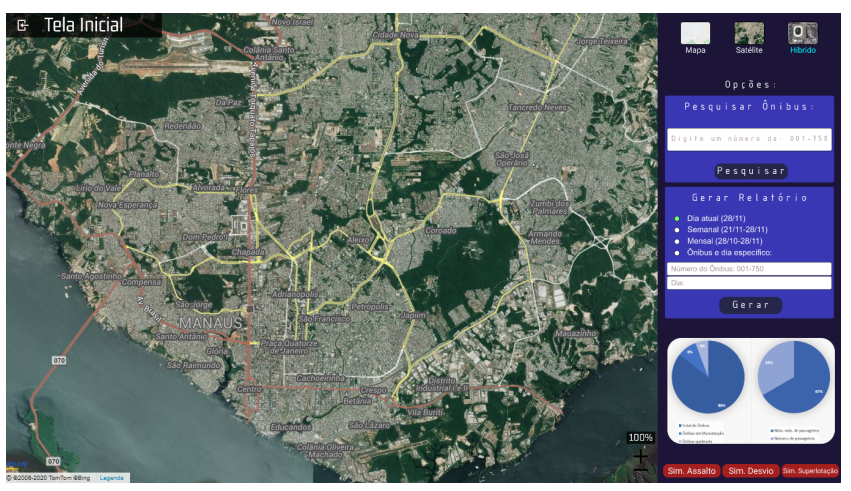

Figura 1: Interface gráfica inicial prototipada no Figma.

Por meio do uso de visão computacional será possível verificar a quantidade de passageiros em um ônibus, dessa forma podendo gerar um melhor controle do fluxo de pessoas. Caso haja em algum momento superlotação em uma linha, o sistema emitirá um alerta para a central de gerenciamento (ver Figura 2).

Um ponto importante a ser tratado é sobre a estrutura do relatório, ou seja, os parâmetros que estarão no template, que são páginas pré-configuradas. Primeiramente será possível gerar quatro tipos de relatórios: diários, semanais, mensais e específicos (por linha e data) para garantir o controle e conhecimento do que ocorre nas linhas de ônibus.

No template também será possível obter as seguintes informações: número da linha dos ônibus; placas dos veículos; carga horária de funcionamento e; caso houver furtos, desvios de rotas, excessos de velocidades ou superlotações, serão mostradas suas respectivas quantidades. Além dessas informações, estará disponível um espaço vazio para a assinatura do monitor e a data.

Com o uso do GPS o sistema poderá detectar possíveis desvios de até $500 \mathrm{~m}$ da rota original, emitindo um alerta (ver Figura 3) para a central de gerenciamento e para a polícia rodoviária. Como o sistema será voltado para o órgão responsável pelo transporte público, as informações serão mais acessíveis e consequentemente, as obras que ocasionarem a mudança das rotas não serão consideradas. 


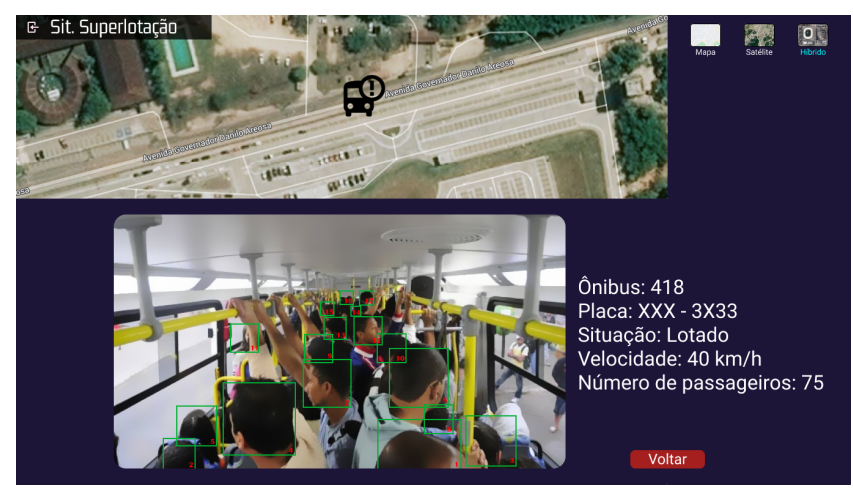

Figura 2: Interface gráfica de alerta de superlotação.

A proposta do sistema é o rastreamento em tempo real, por isso alguns outros métodos não se tornam adequados para tal proposta, como o referenciamento por meio de horários em que o ônibus passará pela parada.

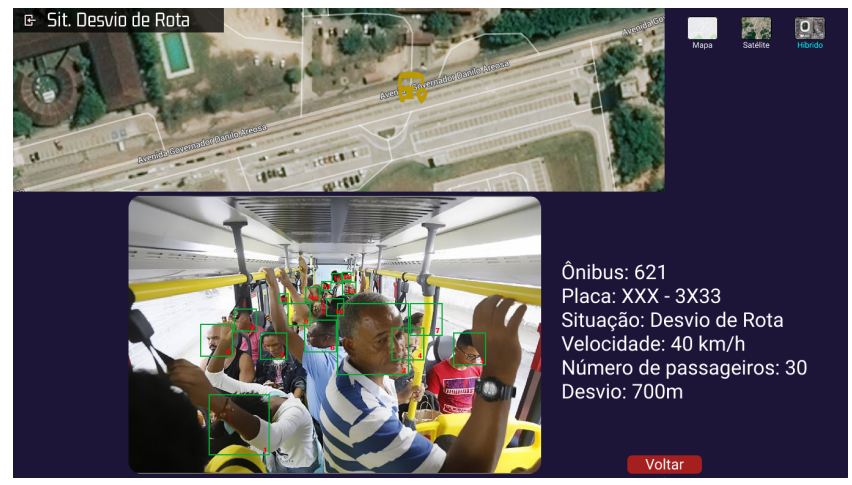

Figura 3: Interface gráfica de alerta de desvios de rotas.

Existem algumas técnicas para detecção de objetos perigosos em ambientes onde se faz necessário a verificação, como raio x e o scanner corporal, porém tais técnicas tem custo muito elevado e operabilidade mais dificultosa para ser usada em um ambiente reduzido como é o caso dos ônibus.

Percebendo essa dificuldade, o sistema usará um método de rastreamento visual baseado na detecção de objetos com visão computacional, que utilizará redes neurais convolucionais capazes de detectar pessoas. O mesmo recurso poderá detectar possíveis artefatos utilizados durante um assalto, como armas de fogo e armas brancas, além de detectar ações potencialmente agressivas com os passageiros ou servidores (motorista, cobrador). O sistema emitirá um alerta (ver Figura 4) para a central de gerenciamento e para a polícia militar.

\section{CONSIDERAÇÕES FINAIS}

Esse artigo teve como objetivo idealizar um sistema inteligente para solucionar problemas que afetam diretamente o transporte público da cidade de Manaus. Para chegar a tal sistema, esse trabalho desenvolveu, com o uso de benchmarking e grupo focal, um protótipo

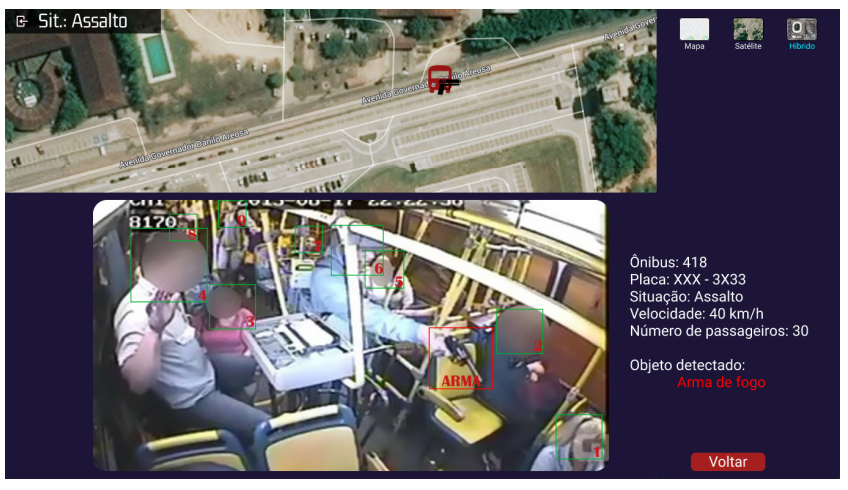

Figura 4: Interface gráfica de alerta de assalto.

de monitoramento em tempo real de alta fidelidade, com o auxílio do software de prototipação gratuito Figma. Como o sistema requer eficiência e maior controle em seus parâmetros, a linguagem $C$ é a melhor escolha por ser uma linguagem que permite a construção do código em alto e baixo nível, com maior portabilidade e manipulação mais profunda sobre o código.

Em relação a visão computacional destacou-se que os métodos como Features de Haar com AdaBoost e descritor HOG são boas soluções para os problemas de detecção de objetos. Portanto, ao utilizar as técnicas propostas no que diz respeito à visão computacional, o sistema terá um melhor controle sobre detecção de rostos e movimentos e consequentemente melhor eficiência para atender aos requisitos.

Caso esse sistema seja desenvolvido futuramente, será possível fazer a detecção do fluxo de passageiros e tentativa de assalto com o uso de visão computacional, como também a verificação da localização em tempo real e desvios de rotas por meio do uso de GPS. Além disso, será possível monitorar a velocidade por meio de sensores e gerar relatórios que serão formulados com os dados obtidos durante o dia, pelo conjunto de funcionalidades do sistema.

\section{AGRADECIMENTOS}

Este trabalho foi realizado com o apoio do Projeto Aranouá, convênio realizado entre o Instituto Federal do Amazonas (IFAM) e a Samsung Eletrônica da Amazônia (SEDA).

\section{REFERÊNCIAS}

[1] Reinaldo Corrêa Costa. Riscos, fragilidades \& problemas ambientais urbanos em manaus. 2017.

[2] Erika Cristine Kneib and Paulo Cesar Marques da Silva. Relação entre demanda e oferta de transporte público coletivo: uma análise estratégica utilizando sig e estatística espacial. Programa de Pós Graduação em Transportes-Doutorado em Transportes, Brasília, 2007.

[3] MR Albertin, H Kohl, and SJB Elias. Manual de benchmarking: um guia para implantação bem-sucedida. Fortaleza: UFC, 2015.

[4] Dirce Stein Backes, Juliana Silveira Colomé, Rolf Herdmann Erdmann, and Valéria Lerch Lunardi. Grupo focal como técnica de coleta e análise de dados em pesquisas qualitativas. O mundo da saúde, 35(4):438-442, 2011.

[5] Herbert Schildt. C completo e total. Makron, 1997.

[6] Ana Caroline Gomes Vargas, Aline Paes, and Cristina Nader Vasconcelos. Um estudo sobre redes neurais convolucionais e sua aplicação em detecção de pedestres. In Proceedings of the xxix conference on graphics, patterns and images, volume 1. sn, 2016.

[7] Gary Bradski and Adrian Kaehler. Opencv. Dr. Dobb's journal of software tools, 3, 2000. 